\title{
Leitlinien der Wissenschaftlichen Medizinischen Fachgesellschaften im Register der AWMF: qualitätsgeprüfte Entscheidungshilfen für die Praxis
}

\section{Zusammenfassung}

Meldungen zu möglichen Einflussnahmen der Pharmaindustrie auf Leitlinien haben die Arbeitsgemeinschaft der Wissenschaftlichen Medizinischen Fachgesellschaften veranlasst, im Rahmen ihrer Delegiertenkonferenz am 09. November 2013 Bilanz zu ziehen über die aktuelle Qualität der Leitlinien im Register der AWMF.

\author{
Ina B. Kopp ${ }^{1}$ \\ Rolf Kreienberg ${ }^{2}$ \\ Wolfgang Müller ${ }^{3}$ \\ 1 AWMF-IMWi \\ 2 AWMF-Leitlinienkommission \\ 3 AWMF-Geschäftsstelle
}

\section{Text}

Die Medizin des 21. Jahrhunderts ist durch einen Kulturwandel geprägt: systematisch entwickelte Leitlinien zur Sicherung der Qualität der medizinischen Versorgung ersetzen die reine Orientierung an Meinungen einzelner Experten, Schulen oder Lehrbüchern. Die Fachgesellschaften erstellen in ehrenamtlichem Engagement hochwertige Leitlinien, die regelmäßig nach dem neuesten Stand des Wissens aktualisiert werden. Als Entscheidungshilfen für Ärzte und Patienten stellen sie diese über das Leitlinienregister der AWMF der Öffentlichkeit kostenfrei zur Verfügung. Für den Inhalt der Leitlinien sind die Fachgesellschaften verantwortlich. Die AWMF unterstützt und berät die Fachgesellschaften, achtet aber auf die Einhaltung strenger Regeln, um die Verlässlichkeit des Leitlinienregisters als Informationsquelle sicher zu stellen. Dazu gehören die öffentliche Ankündigung von Leitlinienvorhaben, die Offenlegung von Interessenkonflikten aller Mitglieder einer Leitliniengruppe und die transparente Darlegung des Entwicklungsprozesses einer Leitlinie. Seit 2010 werden Leitlinien, die nicht alle diese Voraussetzungen erfüllen, nicht mehr in das Register der AWMF aufgenommen. Auch werden Leitlinien, die nicht regelmäßig aktualisiert werden, aus dem Register gelöscht.

Die wichtigsten Qualitätsmerkmale von Leitlinien sind ihre wissenschaftliche Grundlage durch systematische Recherche, Auswahl und Bewertung der Literatur durch Methodiker und Kliniker, der Pluralismus von Interessen durch die für den Adressatenkreis repräsentative Zusammensetzung der Leitliniengruppen und die Vermeidung unerwünschter Beeinflussungen der Gruppendiskussion durch strukturierte Konsensfindungstechniken. Die Erfüllung aller dieser Qualitätsmerkmale wird durch die AWMF sorgfältig geprüft und der jeweiligen Leitlinie eine Klasse zugewiesen, die das Ausmaß der systematischen Entwicklung für den Anwender auf den ersten Blick erkennbar macht (S1-S3). Der Anteil von Leitlinien der höchsten Klasse (S3) nimmt stetig zu. Aktuell enthält das AWMFLeitlinienregister ca. 700 Publikationen, davon entsprechen 123 der Klasse S3 und 159 der Klasse S2.

Die Fachgesellschaften unternehmen erhebliche Anstrengungen, um die inhaltliche Validität der Leitlinien zu gewährleisten. Bei der großen Zahl von Leitlinienempfehlungen kann es aber trotz größter Sorgfalt in Einzelfällen vorkommen, dass Aussagen später korrigiert werden müssen. Dies ist insbesondere dann der Fall, wenn sich nachträglich herausstellt, dass Ergebnisse von Publikationen nicht bestätigt werden. Hinweise auf fehlerhafte Studiengrundlagen sind daher willkommen, werden sehr ernst genommen und von der AWMF an die Fachgesellschaften weitergeleitet. Aktuelle Leitlinien können auch direkt über die AWMF-Internetseite kommentiert werden. Die Fachgesellschaften reagieren schnell - z.B. wurde eine Schmerzleitlinie innerhalb von 4 Wochen nach Bekanntwerden von Studienfälschungen komplett überprüft und ein Addendum publiziert.

Das AWMF-Regelwerk Leitlinien ist online verfügbar: www.awmf.org/leitlinien/awmf-regelwerk.html 


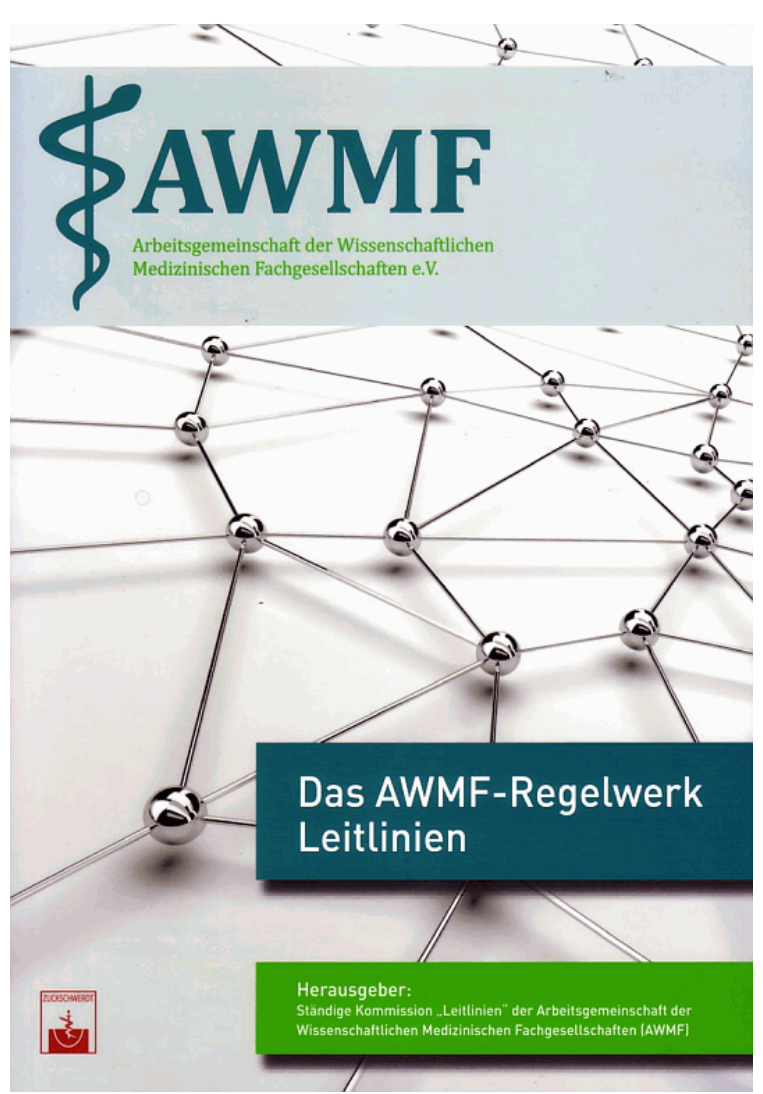

AWMF-Regelwerk Leitlinien

\section{Korrespondenzadresse:}

Prof. Dr. med. Ina B. Kopp

AWMF-IMWi c/o Philipps-Universität, Karl-von-Frisch-Str. 1, 35043 Marburg kopp@awmf.org

\section{Bitte zitieren als}

Kopp I, Kreienberg R, Müller W. Leitlinien der Wissenschaftlichen

Medizinischen Fachgesellschaften im Register der AWMF:

qualitätsgeprüfte Entscheidungshilfen für die Praxis. GMS Mitt AWMF. 2013;10:Doc14.

DOI: 10.3205/awmf000287, URN: urn:nbn:de:0183-awmf0002875

\section{Artikel online frei zugänglich unter}

http://www.egms.de/en/journals/awmf/2013-10/awmf000287.shtml

Eingereicht: 11.11.2013

Veröffentlicht: 12.11.2013

\section{Copyright}

(C)2013 Kopp et al. Dieser Artikel ist ein Open Access-Artikel und steht unter den Creative Commons Lizenzbedingungen

(http://creativecommons.org/licenses/by-nc-nd/3.0/deed.de). Er darf vervielfältigt, verbreitet und öffentlich zugänglich gemacht werden, vorausgesetzt dass Autor und Quelle genannt werden. 